International Journal of Pure and Applied Mathematics

Volume 100 No. 2 2015, 169-192

ISSN: 1311-8080 (printed version); ISSN: 1314-3395 (on-line version)

url: http://www.ijpam.eu

doi: http://dx.doi.org/10.12732/ijpam.v100i2.2

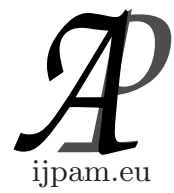

\title{
SECOND ORDER SUFFICIENT OPTIMALITY CONDITIONS \\ FOR SEMILINEAR ELLIPTIC CONTROL PROBLEM OF INFINITE ORDER
}

\author{
S.A. El-Zahaby \\ Department of Mathematics \\ Faculty of Science \\ Al-Azhar University (Girls Branch) \\ Nasr City, Cairo, EGYPT
}

\begin{abstract}
In this paper, we find the necessary conditions for optimality in distributed control problem for semilinear elliptic control problem Governed by elliptic operator of infinite order with finite dimension.

First order and second order sufficient optimality condition are obtained for this control problem using Theorems [14].
\end{abstract}

AMS Subject Classification: 35K20, 49K20, 93C20, 49J20

Key Words: optimal control, elliptic equations, second-order necessary optimality condition, infinite order, semilinear elliptic equations

\section{Introduction}

It is known that in the case of nonlinear equations the first order conditions are not in general sufficient for optimality. In this paper we are going to drive a second order sufficient optimality condition for a class of semilinear elliptic control problems governed by elliptic operator of infinite order with finite dimension.

Received: May 21, 2014

(c) 2015 Academic Publications, Ltd. url: www.acadpubl.eu 
In Dubinskin [5, 6] studied the Cauchy Dirchlet problem

$$
\begin{aligned}
& L(u)=\sum_{|\alpha|=0}^{\infty}(-1)^{|\alpha|} D^{\alpha} A_{\alpha}\left(x, D^{\gamma} u\right)=h(x), \quad x \in G \\
& \left.D^{\omega} u\right|_{\partial G}=0, \quad|\omega|=0,1, \cdots
\end{aligned}
$$

infinite order Sobolev spaces

$$
W^{\infty}\left\{a_{\alpha}, p_{\alpha}\right\}=\left\{u(x) \in C_{0}^{\infty}(G): p(u) \equiv \sum_{|\alpha|=0}^{\infty} a_{\alpha}\left\|D^{\alpha} u\right\|_{p_{a}}^{p_{\alpha}}<\infty\right\},
$$

where $a_{\alpha} \geq 0$ and $p_{\alpha} \geq 1$ are numerical sequences and established the nontriviality of $W^{\infty}\left\{a_{\alpha}, p_{\alpha}\right\}$ and boundary value problem (1) is investigated.

Gali et. al [10] presented a set of inequalities defining an optimal control of a system governed by self-adjoint elliptic operators with an infinite number of variables.

Subsequently, Lions suggested a problem related to this result but in different direction by taking the case of operators of infinite order with finite dimension.

Gali has solved this problem, the result has been published in [9]. Moreover, I. M. Gali et. al $[11,12]$ presented some control problems generated by both elliptic and hyperbolic linear operator of infinite order with finite number of variables.

El-Zahaby et al $[7,8]$ obtained the optimal control of problems governed by variational inequalities of infinite order with bounded domain.

In contrast to the optimal control of linear system with a convex objective where first order necessary optimality conditions are already sufficient for optimality, higher order conditions such as second order sufficient optimality conditions (SSC) should be employed to verify optimality for nonlinear systems.

SSC have also proved to be useful for showing important properties of optimal control problems such as local uniqueness of optimal controls and their stability with respect to certain perturbations. In this respect, we refer to the first investigations of SSC for control problems governed by partial differental equations have been published by Goldberg and Tröltzsch [14].

For elliptic distributed control problems second order sufficient condition were established by Casas and Tröltzsch [2]. It is more or less obvious that these conditions can be transferred by the same technique to elliptic problems of infinite order. 
In the present paper, using the Theory of $[16],[14,15]$ and $[2,3,4]$ we derive the necessary conditions of optimality for control problem governed by semilinear elliptic operator.

First order conditions and the second order sufficient optimality conditions are obtained.

This paper is organized as follows:

- In Section 1, we introduce some functional spaces of infinite order with finite dimension.

- In Section 2, we define a semilinear elliptic control problem generated by elliptic operator of infinite order.

- In Section 3, we derive the first order necessary condition.

- In Section 4, we set up the second order sufficient optimality condition for a semilinear elliptic control problem.

Some Function Spaces: The imbedding problem for non-trivial Sobolev spaces of infinite order is investigated in $[5,6]$. An imbedding criterion is established there in terms of these spaces.

In this case

$$
W^{\infty}\left\{a_{\alpha}, 2\right\} \subseteq L_{2}\left(R^{n}\right) \subseteq W^{-\infty}\left\{a_{\alpha}, 2\right\}
$$

where

$$
W^{\infty}\left\{a_{\alpha}, 2\right\}=\left\{u(x) \in C^{\infty}\left(R^{n}\right): \sum_{|\alpha|=0}^{\infty} a_{\alpha}\left\|D^{\alpha} u\right\|_{2}^{2}<\infty\right\}
$$

are be Soblove spaces of infinite order of periodic function defined on all of $R^{n}$, and $W^{-\infty}\left\{a_{\alpha}, 2\right\}$ denotes their topological dual with respect to $L_{2}\left(R^{n}\right)$. We recall that $\alpha=\left(\alpha_{1}, \alpha_{2}, \cdots, \alpha_{n}\right)$ is a multi-index for differentiation, $|\alpha|=$ $\alpha_{1}+\alpha_{2}+\cdots+\alpha_{n}, D^{\alpha}=\frac{\partial^{|\alpha|}}{\partial x_{1}^{\alpha_{1} \cdots x_{n}^{\alpha_{n}}}}, a_{\alpha} \geq 0$ is a numerical sequence, and $\|\cdot\|_{2}$ is the canonical norm in the space $L_{2}\left(R^{n}\right)$, (all functions are assumed to be real valued).

Let us consider the elliptic operator of infinite order with finite dimension [5]-[9]

$$
A y=\sum_{|\alpha|=0}^{\infty}(-1)^{|\alpha|} a_{\alpha} D^{2 \alpha} y \quad a_{\alpha}>0 .
$$

This operator has a self-adjoint closure. 
We introduce a continuous bilinear form on $W^{\infty}\left\{a_{\alpha}, 2\right\}$

$$
\begin{aligned}
\pi(u, v) & =(A u, v) \\
& =\sum_{|\alpha|=0}^{\infty}\left((-1)^{|\alpha|} a_{\alpha} D^{2 \alpha} u(x), v(x)\right)_{L^{2}\left(R^{n}\right)}, \quad a_{\alpha} \geq 0 \\
& =\sum_{|\alpha|=1}^{\infty}\left((-1)^{|\alpha|} a_{\alpha} D^{2 \alpha} u(x), v(x)\right)_{L^{2}\left(R^{n}\right)}+(q(x) u(x), v(x))_{L_{2}\left(R^{n}\right)}
\end{aligned}
$$

where $q(x)$ is a real valued function from $L_{2}\left(R^{n}\right)$ such that $q(x) \geq \nu, 1 \geq \nu \succ 0$.

The ellipticity of $A$ is sufficient for the coerciveness of $\pi(u, v)$ on $W^{\infty}\left\{a_{\alpha}, 2\right\}$, see $[9]$

In fact

$$
\begin{aligned}
\pi(u, u)= & \sum_{|\alpha|=1}^{\infty} \int_{R^{n}} a_{\alpha}\left(D^{\alpha} u\right)(x)\left(D^{\alpha} u\right)(x) d x+\int_{R^{n}} q(x) u(x) u(x) d x \\
\geq & \sum_{|\alpha|=1}^{\infty} a_{\alpha}\left(\left(D^{\alpha} u\right)(x),\left(D^{\alpha} u\right)(x)\right)_{L_{2}\left(R^{n}\right)}+\nu(u(x), u(x))_{L_{2}\left(R^{n}\right)} \\
= & \sum_{|\alpha|=1}^{\infty} a_{\alpha}\left\|D^{\alpha} u\right\|_{L_{2}\left(R^{n}\right)}^{2}+\nu\|u(x)\|_{L_{2}\left(R^{n}\right)}^{2} \\
= & \sum_{|\alpha|=1}^{\infty} a_{\alpha}\left\|D^{\alpha} u\right\|_{L_{2}\left(R^{n}\right)}^{2}+\nu \sum_{|\alpha|=1}^{\infty} a_{\alpha}\left\|D^{\alpha} u\right\|_{L_{2}\left(R^{n}\right)}^{2} \\
& -\nu \sum_{|\alpha|=1}^{\infty} a_{\alpha}\left\|D^{\alpha} u\right\|_{L_{2}\left(R^{n}\right)}^{2}+\nu\|u\|_{L_{2}\left(R^{n}\right)}^{2} \\
= & \nu\|u\|_{W^{\infty}\left\{a_{\alpha}, 2\right\}}^{2}+(1-\nu) \sum_{|\alpha|=1}^{\infty} a_{\alpha}\left\|D^{\alpha} u\right\|_{L_{2}\left(R^{n}\right)}^{2} .
\end{aligned}
$$

Then

$$
\pi(u, u) \geq \nu\|u\|_{W^{\infty}\left\{a_{\alpha}, 2\right\}}^{2} .
$$




\section{Problem Statement}

Let $\Omega$ be an open and bounded domain in $R^{n}$, with Lipschitz boundary $\Gamma$. In this domain we consider the following state equation

$$
\begin{array}{ll}
A y+d(x, y)=u & \text { in } \Omega \\
\left.y^{|W|}\right|_{\Gamma}=0 & |W|=0,1,2, \cdots \text { on } \Gamma
\end{array}
$$

where $A$ denotes a second order elliptic operator of infinite order having the form (2) in (6) and $d: \Omega \times R \rightarrow R$ is a caratheodory function.

The function $u$ denotes the control in the space of control $L^{2}(\Omega)$ and $y_{u}$ is the solution (state of the function) associated to the control $u$.

\section{Assumption 1:}

(i) Let $\Omega \subset R^{N}$, be a bounded Lipschitz domain with boundary $\Gamma$ and $A$ is an elliptic differential operator of infinite order of the form (2) and with bounded and measurable coefficient and the condition (5) is satisfied, if $q(x) \geq \nu, 1 \geq \nu \succ 0$.

(ii) The function

$$
d(x, y): \Omega \times R \rightarrow R
$$

is bounded and measurable with respect to $x \in \Omega$ for any fixed $y \in R$ and is continuous and monotonously increasing with respect to $y$ for almost all $x$.

\section{Assumption 2:}

It follows from this assumption, in particular, that $d(x, 0)$ is bounded and measurable in $\Omega$, we have a further assumption in view of the problem of unboundedness.

For every $x \in \Omega$ we have $d(x, 0)=0$. Moreover, $d$ is globally bounded, that is, there is a constant $M>0$ such that for any $y \in R$ we have

$$
|d(x, y)| \leq M \quad \text { for a.e } x \in \Omega
$$

The differential equation is associated with the weak formulation of (6)

$$
\begin{aligned}
a[y, v]: & =\sum_{|\alpha|=0}^{\infty} a_{\alpha} \int_{\Omega} D^{\alpha} y(x) D^{\alpha} v(x)+\int_{\Omega} d(x, y) v d x \\
& =\int_{\Omega} u \cdot v d x \quad \forall v \in W^{\infty}\left\{a_{\alpha}, 2\right\}
\end{aligned}
$$


Theorem 1. Suppose that Assumptions 1 and 2 hold. Then the elliptic boundary value problem (6) has a unique solution for all $u \in L^{2}(\Omega)$. If in addition $d(x, 0)=0$, then there exists a constant $C$ independent of $d, u$ so that

$$
\|y\|_{W}{ }_{\left\{a_{\alpha}, 2\right\}} \leq C\|u\| .
$$

Proof. Existence of a solution.

Let us prove that the equation (6)

$$
\begin{array}{ll}
A y+d(x, y(x))=u & \text { in } \Omega \\
\left.y^{|W|}\right|_{\Gamma}=0 & |W|=0,1,2, \cdots \text { on } \Gamma
\end{array}
$$

admits at least one solution $y \in W_{0}^{\infty}\left\{a_{\alpha}, 2\right\}$. We define for fixed $u \in W^{-\infty}\left\{a_{\alpha}, 2\right\}$ $(\Omega)$ and $M>0$, a mapping, $F: z \mapsto y$, where $y \in W^{\infty}\left\{a_{\infty}, 2\right\}$ is the unique solution of the linear equation

$$
\begin{array}{ll}
A y+d(x, z)=u & \text { in } \Omega \\
\left.y^{|W|}\right|_{\Gamma}=0 & |W|=0,1,2, \cdots \text { on } \Gamma
\end{array}
$$

Now, we wanted to show that $F$ has a fixed point, i.e.

$$
F(z)=z
$$

we write the equation (9) as

$$
\begin{array}{ll}
A y=u-d(x, z) & \text { in } \Omega \\
\left.y^{|W|}\right|_{\Gamma}=0 & |W|=0,1,2, \cdots \text { on } \Gamma
\end{array}
$$

Therefore (10) is a linear equation and $u-d(x, z)$ belongs to $W^{-\infty}\left\{a_{\alpha}, 2\right\}$, hence (10) admit a unique solution $y \in W^{\infty}\left\{a_{\alpha}, 2\right\}$ and $F$ is well defined. Thanks to assumption 2 we have $|d(x, y)| \leq M \forall x \in \Omega$ and $y \in R$. By the coerciveness of condition (5) we have

$$
\begin{array}{rlrl}
\|y\|_{W^{\infty}\left\{a_{\alpha}, 2\right\}} & \leq C_{M}\left(\|u\|_{L^{2}(\Omega)}+d\|(x, z)\|_{L^{2}(\Omega)}\right) \\
& \leq r & \forall z \in W^{\infty}\left\{a_{\alpha}, 2\right\}
\end{array}
$$

We can define

$$
C=\left\{z \in W^{\infty}\left\{a_{\alpha}, 2\right\}:\|z\|_{W^{\infty}\left\{a_{\alpha}, 2\right\}} \leq r\right\} \subset W^{\infty}\left\{a_{\alpha}, 2\right\} \subset L^{2}(\Omega)
$$


This set is convex and closed in $W^{\infty}\left\{a_{\alpha}, 2\right\}$ the fact that $W^{\infty}\left\{a_{\alpha}, 2\right\}$ is compactly embedded in $L^{2}(\Omega)$ due to Dubinskii [5].

So consider $F$ as a mapping in $L^{2}(\Omega) F: C \rightarrow C, F$ is continuous. It is easy to apply the Schauder theorem to prove the existence of a fixed point $z \in C$, $F(z)=z$. Obviously, $y$ is a weak solution in $W^{\infty}\left\{a_{\alpha}, 2\right\}$.

Uniqueness of a solution.

Let us assume that $y_{i} \in W^{\infty}\left\{a_{\alpha}, 2\right\}, i=1,2$ are solutions of (6) and satisfy this equation. Subtracting the equations we see that

$$
A\left(y_{1}-y_{2}\right)+d\left(x, y_{1}\right)-d\left(x, y_{2}\right)=u-u=0
$$

multiplying the equation by $\left(y_{1}-y_{2}\right)$ and doing the usual integration by parts we get

$$
\sum_{|\alpha|=0}^{\infty}(-1)^{|\alpha|} \int_{\Omega} a_{\alpha} D^{2 \alpha}\left(y_{1}-y_{2}\right)\left(y_{1}, y_{2}\right) d x+\int_{\Omega}\left(d\left(x, y_{1}\right)-d\left(x, y_{2}\right)\right)\left(y_{1}-y_{2}\right) d x=0
$$

From monotonicity increasing of $d$ with respect to $y$ and the coercivity of the operator $A$ we have

$$
\begin{gathered}
\int_{\Omega}\left(d\left(x, y_{1}\right)-d\left(x, y_{2}\right)\right)\left(y_{1}-y_{2}\right) d x \geq 0 \\
\int_{\Omega} A\left(y_{1}-y_{2}\right)^{2} d x+\int_{\Omega}\left(d\left(x, y_{1}\right)-d\left(x, y_{2}\right)\right)\left(y_{1}-y_{2}\right) d x=0
\end{gathered}
$$

by all are nonnegative and therefore must vanish. Therefore, we have $y_{1}=y_{2}$.

Estimate, let $d(x, 0)=0$. Multiplying the equation (6) by $y$

$$
\begin{gathered}
\sum_{|\alpha|=0}^{\infty} \int_{\Omega} a_{\alpha}\left|D^{\alpha} y\right|^{2} d x+\int_{\Omega} d(x, y) y d x=\int_{\Omega} u y d x \\
\sum_{|\alpha|=0}^{\infty} \int_{\Omega} a_{\alpha}\left|D^{\alpha} y\right|^{2} d x+\int_{\Omega}(d(x, y)-d(x, 0))(y-0) d x=\int_{\Omega} u y d x
\end{gathered}
$$

From the monotonicity increasing we have

$$
\|y\|_{W^{\infty}\left\{a_{\alpha}, 2\right\}} \leq C\|u\|,
$$

and $C$ depends only on the coercivity constant $0<\nu \leq 1$ gives in (5). Indeed $y$ must be continuous. 
We will treat this problem in $W^{\infty}\left\{a_{\alpha}, 2\right\} \cap C(\bar{\Omega})$.

Let $U_{\infty}=L^{\infty}(\Omega)$ and $U_{2}=L_{2}(\Omega)$ be Banach and Hilbert spaces endowed with their national norm $\|\cdot\|_{\infty},\|\cdot\|_{2}$, respectively. We have that $U_{\infty} \subset U_{2}$ with continuous embedding.

We denote by $U_{a d}$ a nonempty convex subset of $U_{\infty}$ that is closed in $U_{2}$. Moreover, an objective function $J: U_{\infty} \rightarrow R$ is given with these quantities. We define the abstract optimization problem

Subject to

$$
\begin{aligned}
& \min _{u \in U_{a d}} J(y, u)=\int_{\Omega} \phi(x, y(x)) d x+\frac{\lambda}{2} \int_{\Omega} u^{2} d x \\
& u_{a}(x) \leq u(x) \leq u_{b}(x) \quad \text { a.e. } x \in \Omega
\end{aligned}
$$

$$
\begin{aligned}
& A y+d(x, y)=u \quad \text { in } \Omega \\
& \left.y^{|W|}\right|_{\Gamma}=0
\end{aligned}
$$

where $A$ is infinite order operator with finite dimension of the form (2)

We recall that problems in which the control occurs as a source term on the right hand side of the partial differential equations are termed distributed control problems. Here, the set of admissible control is given by

$$
U_{a d}=\left\{u \in L^{2}(\Omega): a_{\alpha}(x) \leq u(x) \leq u_{b}(x) \text { for a.e. } x \in \Omega\right\}
$$

$U_{a d}$ is bounded, convex and closed subset of $L_{2}(\Omega)$.

Definition 2. A control $\bar{u} \in U_{a d}$ is said to be optimal if it satisfies, together with the associated optimal state $\hat{y}=y(\bar{u})$, the inequality

$$
J(y(\bar{u}), \bar{u}) \leq J(y(u), u) \quad \forall \quad u \in U_{a d}
$$

A control is said to be locally optimal in the sense of $L^{2}(\Omega)$ if there exists some $\epsilon>0$ such that the above inequality holds for all $u \in U_{a d}$ such that $\|u-\bar{u}\|_{L^{2}(\Omega)}<\epsilon$.

Before stating the first result on the existence of optimal controls we note two properties of the functionals

$$
\begin{aligned}
& F(y)=\int \phi(x, y(x)) d x, \\
& Q(u)=\frac{\lambda}{2} \int_{\Omega} u^{2} d x
\end{aligned}
$$

The functionals are composed of Nemytskii operators and a continuous linear integral operator from $L^{1}(\Omega)$ into $R$. Such that these operator are differentiable in $L^{\infty}(\Omega), u \in L^{2}(\Omega)$. By virtue of (Lemma 4.11 on page 198 [16]) $F$ is Lipschitz continuous on the set $\left\{y \in L^{2}(\Omega):\|y\|_{L^{\infty}(\Omega)} \leq M\right\}$ 


\section{Assumption 3}

(i) $\Omega \subset R^{N}$ is a bounded Lipschitz domain.

(ii) The function $d=d(x, y), \phi(x, y): \Omega \times R \rightarrow R$, where $E=\Omega$ are measurable with respect to $x$ for every $y \in R$ and twice differentiable with respect to $y$ (respectively $u$ ) for almost every $x \in \Omega$. Moreover, they satisfy the boundedness and local Lipschitz condition of order $k=2$ that for $\phi$ this means for example, that there are constant $k>0$ and $L(M)>0$ such that for almost every $x \in \Omega$ we have

$$
\begin{gathered}
|\phi(x, 0)|+\left|\phi_{y}(x, 0)\right|+\left|\phi_{y y}(x, 0)\right| \leq K \\
\left|\phi_{y y}\left(x, y_{1}\right)-\phi_{y y}\left(x, y_{2}\right)\right| \leq L(M)\left|y_{1}-y_{2}\right| \quad \forall \quad y_{1}, y_{2} \in[-M, M]
\end{gathered}
$$

(iii) Additionally, $d_{y}(x, y) \geq 0$ for almost every $x \in \Omega$ and all $y \in R$. Moreover, there is a set $E_{d} \subset \Omega$ of positive measure and constant $\lambda_{d}>0$ such that

$$
d_{y}(x, y) \geq \lambda_{d} \quad \forall x \in E_{d}, \quad \forall \quad y \in R
$$

(iv) The bounds $u_{a}, u_{b}: \Omega \rightarrow R$ belong to $L^{\infty}(\Omega)$ and satisfy the condition $u_{a}(x) \leq u_{b}(x)$ for almost every $x \in \Omega$.

As mentioned [14], the above set of assumptions is too restrictive for the existence proof. In fact, for the existence of optimal controls the conditions in (ii) concerning the derivations of $\phi$ and $\psi$ are dispensable, these conditions including Lipschitz continuity, are needed only for the functions themselves (order $k=0$ ). On the other hand we have to postulate that $Q(u)$ is convex with respect to $u$.

Remark 3. To prove the existence of optimal control, we only Need part (ii) for the function $\phi$ but not for their derivatives. For the first-order optimality conditions, the conditions for the second-order derivatives are not needed. While Assumption 3 is needed in its entirety for second order conditions.

Let $G$ be the mapping

$$
G: L^{2}(\Omega) \rightarrow C(\bar{\Omega}) \cap W^{\infty}\left\{a_{\alpha}, 2\right\},
$$

the solution mapping $G:=u \mapsto y$, the solution $y=G(u)$. Obviously $G$ is a nonlinear mapping also continuous and the problem $(\mathrm{P})$ is become

$$
\begin{aligned}
\min \quad f(u) & =J(y, u)=J(G(u), u) \\
& =\int_{\Omega} \phi(x, G(u)(x)) d x+\frac{\lambda}{2} \int_{\Omega} u^{2}(x) d x
\end{aligned}
$$


The problem is to find

$$
\min _{u \in U_{a d}} F(u)+Q(u)
$$

where

$$
U_{a d}=\left\{u \in L^{2}(\Omega): u_{a}(x) \leq u(x) \leq u_{b}(x)\right\}
$$

Theorem 4. Suppose that Assumption 3 holds for $\Omega$ and $d$. Then $G$ is a Lepschitz continuous mapping from $L^{2}(\Omega)$ into $W^{\infty}\left\{a_{\alpha}, 2\right\} \cap C(\bar{\Omega})$, that, there is a constant $L>0$ such that

$$
\left\|G\left(u_{1}\right)-G\left(u_{2}\right)\right\| \leq L\left\|u_{1}-u_{2}\right\|_{L^{2}(\Omega)}
$$

i.e.

$$
\left\|y_{1}-y_{2}\right\|_{W^{\infty}\left\{a_{\alpha}, 2\right\}}+\left\|y_{1}-y_{2}\right\|_{C(\bar{\Omega})} \leq L\left\|u_{1}-u_{2}\right\|_{L^{2}(\Omega)}
$$

wherever $u_{i} \in L^{2}(\Omega)$ and $y_{i}=G\left(u_{i}\right), i=1,2$.

Proof. By Theorem 1, $y_{i} \in C(\bar{\Omega})$ for $i=1,2$ in equation (6). We see that

$$
\begin{aligned}
& A y_{1}+d\left(x, y_{1}\right)=u_{1}, \\
& A y_{2}+d\left(x, y_{2}\right)=u_{2} .
\end{aligned}
$$

Subtracting the equations satisfied by $y_{1}$ and $y_{2}$

$$
\begin{aligned}
& A\left(y_{1}-y_{2}\right)+d\left(x, y_{1}\right)-d\left(x, y_{2}\right)=u_{1}-u_{2} \\
& \left.\left(y_{1}-y_{2}\right)^{|W|}\right|_{\Gamma}=0, \quad|W|=0,1,2, \cdots
\end{aligned}
$$

Evidently, we have by the mean value theorem

$$
\begin{aligned}
d\left(x, y_{1}(x)\right)-d\left(x, y_{2}(x)\right) & =-\int_{\Omega} \frac{d}{d s} d\left(x, y_{1}(x)+s\left(y_{2}(x)-y_{1}(x)\right)\right) d s \\
& =\int_{\Omega} d_{y}\left(x, y_{1}(x)+s\left(y_{2}(x)-y_{1}(x)\right)\right) d s\left(y_{1}(x)-y_{2}(x)\right)
\end{aligned}
$$

according to the continuity of the functions $d_{y}, y_{1}$ and $y_{2}$ the integral on $\Omega$ the integral satisfies

$$
d_{y}\left(x, y_{1}(x)+s\left(y_{2}(x)-y_{1}(x)\right) \geq \lambda_{d}>0\right.
$$

According to the continuity of the function $d_{y}, y_{1}, y_{2}$. The integral in the second terms is nonnegative science the mapping $y \rightarrow d(x, y)$ is monotone increasing. The integral satisfies

$$
d_{y}\left(x, y_{1}(x)\right)+s\left(y_{2}(x)-y_{1}(x)\right)=\beta(x)\left(y_{1}(x)-y_{2}(x)\right) \text { and by } d_{y} \geq 0, \beta \geq 0
$$




$$
\begin{aligned}
& A\left(y_{1}-y_{2}\right)+\beta\left(y_{1}-y_{2}\right)=u_{1}-u_{2} \\
& \left.\left(y_{1}-y_{2}\right)^{|W|}\right|_{\Gamma}=0
\end{aligned}
$$

as in linear Theory, now it follows $\exists L>0$

$$
\left\|y_{1}-y_{2}\right\|_{W^{\infty}\left\{a_{\alpha}, 2\right\}}+\left\|y_{1}-y_{2}\right\|_{C(\bar{\Omega})} \leq L\left\|u_{1}-u_{2}\right\|_{L^{2}(\Omega)} .
$$

Theorem 5. Suppose that Assumption 3 holds, the problem (P) has at least one optimal control $\bar{u}$.

Proof. The set

$$
U_{a d}=\left\{u \in L^{2}(\Omega): u_{a} \leq u \leq u_{b}\right\}
$$

is bounded in $L^{\infty}(\Omega)$ and thus bounded in the space $L^{2}(\Omega)$.

The set of the associated solutions $y$ is bounded in $W^{\infty}\left\{a_{\alpha}, 2\right\} \cap C(\bar{\Omega})$. By Assumption 3 the cost functional $J(y, u)$ is continuous. Therefore, by boundedness from below, the infimum

$$
j=\inf _{u \in U_{\text {ad }}} J(y(u), u)=\inf _{u \in U_{a d}} J(G(u), u)
$$

exists and a sequence

$$
\left\{u_{n}\right\} \subset U_{a d}, \quad y_{n}=G\left(u_{n}\right)
$$

for $n \in N$ such that

$$
J\left(y_{n}, u_{n}\right) \rightarrow j \quad \text { as } \quad n \rightarrow \infty .
$$

$U_{a d}$ is nonempty, closed, bounded and convex in $L^{2}(\Omega)$. That is $U_{a d}$ weakly sequentially compact. Hence there exists a subsequence $u_{n_{k}} \rightarrow \bar{u}$ weakly, $k \rightarrow$ $\infty$ without loss of generality $\left\{u_{n}\right\}_{n=1}^{\infty}$ itself, $u_{n} \rightarrow \bar{u}$ as $n \rightarrow \infty$.

Next, observe that $y_{n}$ solves the equation

$$
A y_{n}+d\left(x, y_{n}\right)=u_{n}
$$

$\left\{u_{n}\right\}$ is bounded in $W^{\infty}\left\{a_{\alpha}, 2\right\}$ and in $C(\bar{\Omega})$.

$W^{\infty}\left\{a_{\alpha}, 2\right\}$ is compactly imbedded in $L^{2}(\Omega)$ (See Dubinskii $[5,6]$ ), we can assume

$$
\begin{array}{ll}
y_{n} \rightarrow \bar{y} & \text { in } \quad L^{2}(\Omega) \\
d\left(x, y_{n}\right) \rightarrow z & \text { in } \quad L^{2}(\Omega)
\end{array}
$$


However, $y_{n}$ is bounded in $C(\bar{\Omega})$

$$
\left\|y_{n}\right\|_{C(\bar{\Omega})} \leq M \quad \forall \quad n \in N
$$

From

$$
u_{n} \rightarrow \bar{u} \quad \text { in } \quad L^{2}(\Omega)
$$

and $y_{n} \rightarrow \bar{y}$ in $W^{\infty}\left\{a_{\alpha}, 2\right\}$ yields the convergence of the integral.

$$
\sum_{|\alpha|=0}^{\infty} a_{\alpha} \int_{\Omega} D^{\alpha} y_{n} D^{\alpha} v d x+\int_{\Omega} d\left(., y_{n}\right) v d x=\int u_{n} v d x \quad \forall v \in W^{\infty}\left\{a_{\alpha}, 2\right\}
$$

we have

$$
\sum_{|\alpha|=0}^{\infty} a_{\alpha} \int_{\omega} D^{\alpha} y D^{\alpha} v d x+\int d(., \bar{y}(x)) v d x=\int_{\omega} \bar{u} v d x
$$

In other words $\bar{y}$ is the weak solution corresponding to the right hand side $\bar{u}$ that is $\bar{y}=y(\bar{u})$.

The functional $Q$ already suffices to conclude from the convergence $u_{n} \rightarrow \bar{u}$ that $Q\left(u_{n}\right) \rightarrow Q(\bar{u})$.

Hence by convexity the functional $Q$ is therefore weakly lower semicontinuous that is

$$
u_{n} \rightarrow \bar{u} \Rightarrow \lim _{n \rightarrow \infty} \inf Q\left(u_{n}\right) \geq Q(\bar{u})
$$

In summary we have

$$
\begin{aligned}
j & =\lim _{n \rightarrow \infty} J\left(y_{n}, u_{n}\right) \\
& =\lim _{n \rightarrow \infty} F\left(y_{n}\right)+\lim _{n \rightarrow \infty} \inf Q\left(u_{n}\right) \\
& =F(\bar{y})+\lim _{n \rightarrow \infty} \inf Q\left(u_{n}\right) \\
& \geq F(\bar{y})+Q(\bar{u}) \\
& =J(\bar{y}, \bar{u}) .
\end{aligned}
$$

By definition of the infimum $j$, we therefore must have

$$
J(\bar{y}, \bar{u})=j,
$$

which proves the optimality.

Next, we determine the Frechet derivative of the control-to-state operator at a first point $\bar{u}$. 
Theorem 6. Suppose that the Assumption 3 holds. Then for every $r>\frac{N}{2}$ the control to state operators $G$ is Frechet differentiable from $L^{2}(\Omega)$ into $W^{\infty}\left\{a_{\alpha}, 2\right\} \cap C(\bar{\Omega})$. The directional derivative at $\bar{u} \in L^{2}(\Omega)$ in the direction $u$ is given by

$$
G^{\prime}(\bar{u}) u=y,
$$

where $y$ denotes the weak solution to the boundary value problem linearized at $\bar{y}=G(\bar{u}):$

$$
\begin{array}{lc}
A y+d_{y}(x, \bar{y}) y=u & \text { in } \quad \Omega \\
\left.y^{|W|}\right|_{\Gamma}=0, & |W|=0,1,2, \cdots
\end{array}
$$

Proof. We have to show that

$$
G(\bar{u}+u)-G(\bar{u})=D u+r(\bar{u}, u)
$$

with a continuous linear operator

$$
D: L^{2}(\Omega) \rightarrow W^{\infty}\left\{a_{\alpha}, 2\right\} \cap C(\bar{\Omega})
$$

and a mapping $r$ that satisfies

$$
\frac{\|r(\bar{u}, u)\|_{W^{\infty}\left\{a_{\alpha}, 2\right\} \cap C(\bar{\Omega})}}{\|u\|_{L^{2}(\Omega)}} \rightarrow 0 \quad \text { as } \quad\|u\|_{L^{2}(\Omega)} \rightarrow 0
$$

Here we put

$$
\|r\|_{W^{\infty}\left\{a_{\alpha}, 2\right\} \cap C(\bar{\Omega})}:=\|r\|_{W^{\infty}\left\{a_{\alpha}, 2\right\}}+\|r\|_{C(\bar{\Omega})}
$$

It then follows that

$$
G^{\prime}(\bar{u})=D
$$

The boundary value problems satisfied by $\bar{y}=y(\bar{u})$ and $\tilde{y}=y(\bar{u}+u)$, read respectively,

$$
\begin{array}{ll}
A \bar{y}+d(x, \bar{y})=\bar{u} & \\
\left.y^{|W|}\right|_{\Gamma}=0 & |W|=0,1,2, \cdots \\
A \tilde{y}+d(x, \tilde{y})= & \bar{u}+u \\
\left.y^{|W|}\right|_{\Gamma}=0, & |W|=0,1,2, \cdots
\end{array}
$$


subtracting them yields

$$
\begin{gathered}
d_{y}(x, \bar{y})(\tilde{y}-\bar{y})+r_{d} \\
A(\tilde{y}-\bar{y})+\overbrace{d(x, \tilde{y})-d(x, \bar{y})}=u \\
\left.(\tilde{y}-\bar{y})^{|W|}\right|_{\Gamma}=0, \quad|W|=0,1,2, \cdots
\end{gathered}
$$

The Nemytskii operator $d(., y()$.$) is Frechet differentiable from C(\bar{\Omega})$ into $L^{\infty}(\Omega)$ see $[16]$,

$$
d(., \tilde{y}(.))-d(., \bar{y}(.))=d_{y}(., \bar{y}(.))(\tilde{y}(.)-\bar{y}(.))+r_{d}
$$

with a remainder $r_{d}$ such that

$$
\frac{\left\|r_{d}\right\|_{L^{\infty}(\Omega)}}{\|\tilde{y}-\bar{y}\|_{C(\bar{\Omega})}} \rightarrow 0 \quad \text { as } \quad\|\tilde{y}-\bar{y}\|_{C(\bar{\Omega})} \rightarrow 0
$$

this implies that

$$
\tilde{y}-\bar{y}=y+y_{\rho}
$$

with the solution $y$ to $(2.8)$ and a reminder $y_{\rho}$ that solves the boundary value problem

$$
\begin{aligned}
& A y_{\rho}+d_{y}(., \bar{y}) y_{\rho}=-r_{d} \\
& \left.y_{\rho}^{|W|}\right|_{\Gamma}=0, \quad|W|=0,1, \cdots
\end{aligned}
$$

In this connection, recall that $d_{y}(x, \bar{y}) \geq \lambda_{d}>0$ in $\Omega$ so that this problem is uniquely solvable.

From the Lipschitz continuity shown in Theorem 2.3 it follows that

$$
\|\tilde{y}-\bar{y}\|_{C(\bar{\Omega})}+\|\tilde{y}-\bar{y}\|_{W^{|\infty|}\left\{a_{\alpha}, 2\right\}} \leq L\|u\|_{L^{2}(\Omega)}
$$

Moreover,

$$
\begin{aligned}
\frac{\left\|r_{d}\right\|_{L^{\infty}(\Omega)}}{\|u\|_{L^{2}(\Omega)}} & =\frac{\left\|r_{d}\right\|_{L^{\infty}(\Omega)}}{\|\tilde{y}-\bar{y}\|_{C}(\bar{\Omega})} \frac{\|\tilde{y}-\bar{y}\|_{C}(\bar{\Omega})}{\|u\|_{L^{2}(\Omega)}} \\
& \leq \frac{\left\|r_{d}\right\|_{L^{\infty}(\Omega)}}{\|\tilde{y}-\bar{y}\|_{C}(\bar{\Omega})} L .
\end{aligned}
$$

and thus

$$
\left\|r_{d}\right\|_{L^{\infty}(\Omega)}=o\left(\|u\|_{L^{2}(\Omega)}\right)
$$


by (14) we also have

$$
\left\|y_{\rho}\right\|_{C(\bar{\Omega})}+\left\|y_{p}\right\|_{W|\infty|\left\{a_{\alpha}, 2\right\}}=o\left(\|u\|_{L^{2}(\Omega)}\right)
$$

Denoting the continuous linear mapping $u \rightarrow y$ by $D$, we conclude that

$$
\begin{aligned}
G(\bar{u}+u)-G(\bar{u}) & =\tilde{y}-\bar{y} \\
& =D u+y_{\rho} \\
& =D u+r(\bar{u}, u),
\end{aligned}
$$

where $r(\bar{u}, u)=y_{\rho}$ has the required properties.

Conclusion: $G$ is continuous Fréchet differentiable from $L^{2}(\Omega)$ to $W^{\infty}\left\{a_{\alpha}, 2\right\} \cap$ $C(\bar{\Omega})$. The derivative $G^{\prime}(\bar{u}) u$ at $\bar{y}$ is $y$ where $y$ denotes the weak solution to the boundary value problem linearized at $\bar{y}=G(\bar{u})$ that is

$$
\begin{aligned}
& A y+d_{y}(x, \bar{y}) y=u \\
& \left.y^{|W|}\right|_{\Gamma}=0, \quad|W|=0,1,2, \cdots
\end{aligned}
$$

\section{Necessary Optimality Conditions}

We derive the first order necessary condition that have to be obeyed by $\bar{u}$ and the associated state $\bar{y}$.

Let $\bar{u}$ be locally optimal in the space of $U=L^{2}(\Omega)$ with associated state $\bar{y}=G(\bar{u})$. The cost functional is

$$
\begin{aligned}
J(y, u) & =J(G(u), u) \\
& =F(G(u))+Q(u) \\
& =: f(u)
\end{aligned}
$$

where $F$ and $Q$ are defined as in (11) and under our Assumption $3 f$ is a Fréchet differentiable function in $L^{\infty}(\Omega)$, indeed $F, G$ and $G$ are Frechet differentiable.

Under Assumption 3, let $\bar{u}$ be a locally optimal control for the problem (P). Then we have the variational inequality

$$
f^{\prime}(\bar{u})(u-\bar{u}) \geq 0 \quad \forall \quad u \in U_{a d} .
$$


Theorem 7. Suppose that Assumption 3 holds. Then every locally optimal control $\bar{u}$ for problem $(P)$ satisfies together with the associated adjoint state $p \in W^{\infty}\left\{a_{\alpha}, 2\right\} \cap C(\bar{\Omega})$ defined by (17) the variational inequality.

$$
\int_{\Omega}(p(x)+\lambda(\bar{u}(x))(u(x)-\bar{u}(x))) d x \geq 0 \quad \forall u \in U_{a d}
$$

Proof. The above result (15) is valid for all nonlinear functional of the type

$$
\begin{aligned}
f(u) & =J(y(x), u) \\
& =J(G(u), u) \\
& =F(G(u), u) .
\end{aligned}
$$

Using the chain rule, we immediately see that the directional derivative at $\bar{u}$ in the direction $h$ is given by

$$
\begin{aligned}
f^{\prime}(\bar{u}) h & =F^{\prime}(G(\bar{u})) G^{\prime}(\bar{u}) h+Q^{\prime}(\bar{u}) h \\
& =F^{\prime}(\bar{y}) y+Q^{\prime}(\bar{u}) h \\
& =\int_{\Omega} \phi_{y}(x, \bar{y}(x)) y(x) d x+\int_{\Omega} \psi_{u}(x, \bar{u}(x)) h(x) d x \\
& =\int_{\Omega} \phi_{y}(x, \bar{y}(x)) y(x) d x+\lambda \int_{\Omega} u h d x .
\end{aligned}
$$

Here $y=G^{\prime}(\bar{u}) h$ by Theorem 2.4 is the weak solution to the linearized boundary value problem.

$$
\begin{aligned}
& A y+d_{y}(x, \bar{y}) y=h \\
& \left.y^{|W|}\right|_{\Gamma}=0
\end{aligned}
$$

Next, we define the adjoint state $p \in W^{\infty}\left\{a_{\alpha}, 2\right\} \cap C(\bar{\Omega})$ as the unique weak solution to the adjoint solution to the adjoint equation

$$
\begin{aligned}
& A p+d_{y}(x, \bar{y}) p=\phi_{y}(x, \bar{y}(x)) \quad \text { in } \Omega \\
& \left.p^{|W|}\right|_{\Gamma}=0, \quad|W|=0,1,2, \cdots
\end{aligned}
$$

Let $y$ be the weak solution to problem (16). Now choosing $p$ as test function in the weak formulation of (16), we obtain

$$
\begin{aligned}
& \sum_{|\alpha|=1}^{\infty} \int_{\Omega} a_{\alpha}\left(D^{\alpha} y\right)(x)\left(D^{\alpha} p\right)(x) d x+\int_{\Omega} q(x) y(x) p(x) d x \\
& \quad+\int_{\Omega} d_{y}(x, \bar{y}) p(x) y d x=\int_{\Omega} h p(x) d x
\end{aligned}
$$


on the other hand we insert $y$ in the weak formulation of the equation (17)

$$
\begin{aligned}
& \sum_{|\alpha|=1}^{\infty} \int_{\Omega} a_{\alpha}\left(D^{\alpha} y\right)(x)\left(D^{\alpha} p\right)(x) d x+\int_{\Omega} q(x) y(x) p(x) d x \\
& \quad+\int_{\Omega} d_{y}(x, y) p y=\int_{\Omega} \phi_{y}(x, \bar{y}(x)) y(x) d x
\end{aligned}
$$

subtracting one equation from the other finally yields

$$
\int_{\Omega} \phi_{y}(x, \bar{y}) y(x) d x=\int_{\Omega} p(x) h(x) d x
$$

As a simple conclusion, the following expression for the directional derivative of the reduced functional $f$ at $\bar{u}$ in the direction $h \in L^{\infty}(\Omega)$ results:

$$
f^{\prime}(\bar{u}) h=\int_{\Omega}(p(x)+\lambda \bar{u}) h(x) d x, h=u-\bar{u}
$$

We obtain the desired necessary optimality condition.

Now, we can reformulate the variational inequality in terms of a minimum principle

$$
\min _{u_{a}(x) \leq v \leq u_{b}(x)}\{p(x)+\lambda \bar{u}(x) v\}
$$

is attained at $v=\bar{u}$ for almost every $x \in \Omega$.

Therefore, we have the projection formula

$$
\bar{u}(x)=P_{\left[u_{a}(x), u_{b}(x)\right]}\left\{-\frac{1}{\lambda} p(x)\right\} \quad \text { for a.e. } x \in \Omega, \quad \text { see }[16] .
$$

If $u_{a}$ and $u_{b}$ are continuous, then so is $\bar{u}$, in fact, we have $p \in W^{\infty}\left\{a_{\alpha}, 2\right\}(\Omega) \cap$ $C(\bar{\Omega})$ and the projection operator maps conditions functions to continuous ones.

The optimality system is

$$
\begin{aligned}
& A y+d(x, y)=P_{\left[u_{\alpha}(x), u_{\beta}(x)\right]}\left\{-\frac{1}{\lambda} p(x)\right\} \quad \text { for a.e. } x \in \Omega \\
& \begin{array}{l}
y^{|W|}=0 \quad \text { on } \quad \Omega \\
A p+d_{y}(x, y) p=\phi_{y}(x, \bar{y}) \quad \text { in } \quad \Omega
\end{array} \\
& \left.p^{|W|}\right|_{\Omega}=0
\end{aligned}
$$




\section{Second Order Derivatives}

As before, we denote by $G$ the control to state mapping $u \rightarrow y$ for the above elliptic boundary value problem. We consider $G$ as a mapping between $L^{2}(\Omega)$ and $W^{\infty}\left\{a_{\alpha}, 2\right\} \cap C(\bar{\Omega})$. First, we show existence and continuity of the secondorder Fréchet derivative of $G$.

Theorem 8. Suppose that Assumption 3 holds. then the operators $G$ : $L^{2}(\Omega) \rightarrow W^{\infty}\left\{a_{\alpha}, 2\right\} \cap C(\bar{\Omega})$ is twice continuity Fréchet differentiable. The second derivative $G^{\prime \prime}(u)$ is given by

$$
G^{\prime \prime}(u)\left[u_{1}, u_{2}\right]=z
$$

where $z$ is a unique weak solution to the elliptic boundary value problem

$$
A z+d_{y}(x, y) z=-d_{y y}(x, y) y_{1} y_{2}
$$

where $y=G(u)$ and $y_{i}=G^{\prime}(u) u_{i} \in W^{\infty}\left\{a_{\alpha}, 2\right\}$ for $i=1,2$.

Proof. (i) Existence of the second derivative. By Theorem (2.4), we know $G$ is Fréchet differentiable. To show the existence of the second derivative, we apply the implicit function Theorem. To this end we transform the elliptic boundary value problem for $y=G(u)$ into a suitable form. For this purpose, let $R: L^{\infty}(\Omega) \rightarrow W^{\infty}\left\{a_{\alpha}, 2\right\} \cap C(\bar{\Omega})$ denote the solution operator of the linear elliptic boundary value problem

$$
\begin{array}{cc}
A y+y=v & \text { in } \Omega \\
y^{|W|} \mid \Gamma=0, & |W|=0,1,2, \cdots
\end{array}
$$

where $A$ is a operators of infinite order has the form (2). We regard $R$ as an operator with range in $C(\bar{\Omega})$. The equation $y=G(u)$ means that

$$
\begin{aligned}
& A y+y=u-d(x, y)+y \quad \text { in } \Omega \\
& \left.y^{|W|}\right|_{\Gamma}=0, \quad|W|=0,1,2, \cdots
\end{aligned}
$$

In terms of $R$ this means that $y=R(u-d(., y)+y)$ or more precisely,

$$
y-R(u-\Phi(y))=0
$$

where $\Phi: C(\bar{\Omega}) \rightarrow L^{\infty}(\Omega)$ denote the Nemytskeii operator generated by $d(., y)-y$. Obviously, if $y \in C(\bar{\Omega})$ solve $(20)$, then $y$ automatically lies in 
the range of $R$ and hence belongs to $W^{\infty}\left\{a_{\alpha}, 2\right\}$ and is a weak solution to $(19)$.

Therefore (19) and (20) are equivalent.

Next, we define the operator $F: C(\bar{\Omega}) \times L^{\infty}(\Omega) \rightarrow C(\bar{\Omega})$

$$
F(y, u)=y-R(u-\Phi(y)) .
$$

Since $\phi$ is, twice continuously differentiable and $R$ is linear and continuous, it follows from the chain rule that $F$ is also a twice continuously Fréchet differentiable.

Moreover, the derivative $D_{y} F(y, u)$ is surjective in fact, the equality

$$
D_{y} F(y, u) w=v
$$

is equivalent to $w+R \Phi^{\prime}(y) w=v$ and putting $\tilde{w}=w-v$, to

$$
\tilde{w}=-R \Phi^{\prime}(y)(\tilde{w}+v) .
$$

A straight forward calculation, using the definition of $R$, whom that the latter equation is equivalent to the boundary value problem

$$
\begin{aligned}
& A \tilde{w}+d_{y}(x, y) \tilde{w}=-d_{y}(x, y) v+v \\
& \left.\tilde{w}^{|W|}\right|_{\Omega}=0, \quad|W|=0,1,2 \cdots
\end{aligned}
$$

which for every $v \in C(\bar{\Omega})$ has a unique solutions $\tilde{w} \in W^{\infty}\left\{a_{\alpha}, 2\right\} \cap C(\bar{\Omega})$.

In summary, all assumptions of the implicit function are satisfied and therefore, the equation $F(y, u)=0$ has a unique solution $y=G(u)$ for any $u$ in a suitable open neighborhood of $\bar{u}$. We have shown already that a unique solution $y=G(u)$ exists even for all $u \in U_{a d}$.

However, the implicit function Theorem also yields that $G$ inherits the smoothness properties of $F$ see, e.g. [Car 1]; therefore, $G$ is twice continuously Fréchet differentiable.

(ii) Calculation of $G^{\prime \prime}(u)$.

Taking $y=G(u)$ in the definition of $F$, we see that

$$
F(G(u), u)=G(u)-R u+R \Phi(G(u))=0
$$

Differentiation in the direction $u_{1}$ yields, by the chain rule,

$$
G^{\prime}(u) u_{1}-R u_{1}+R \Phi^{\prime}(G(u)) G^{\prime}(u) u_{1}=0 .
$$


We define the operator $K: L^{\infty}(\Omega) \rightarrow C(\bar{\Omega}), K(u):=G^{\prime}(u) u_{1}$,

$$
K(u)-R u_{1}+R \Phi^{\prime}(G(u)) K(u)=0
$$

Next, we calculate the directional derivative in the direction $u_{2}$, using the product and chain rules, we obtain that

$$
K^{\prime}(u) u_{2}+R \Phi^{\prime \prime}(G(u))\left[K(u), G^{\prime}(u) u_{2}\right]+R \phi^{\prime}(G(u)) k^{\prime}(u) u_{2}=0 .
$$

Since

$$
K^{\prime}(u) u_{2}=G^{\prime \prime}(u)\left[u_{1}, u_{2}\right]
$$

(cf.the scheme for the evaluation of second order derivative explained on page $227[16])$.

This is turn equivalent to the equation

$$
G^{\prime \prime}(u)\left[u_{1}, u_{2}\right]+R \Phi^{\prime \prime}(G(u))\left[G^{\prime}(u) u_{1}, G^{\prime}(u) u_{2}\right]+R \Phi^{\prime}(G(u)) G^{\prime \prime}(u)\left[u_{1}, u_{2}\right]=0
$$

with $z:=G^{\prime \prime}(u)\left[u_{1}, u_{2}\right]$, we thus have

$$
z+R\left(\Phi^{\prime}(y) z+\Phi^{\prime \prime}(y)\left[y_{1}, y_{2}\right]\right)=0
$$

which by the definition of $R$ means that $z$ is the unique solution to the boundary value problem

$$
\begin{aligned}
& A z=-d_{y}(x, y) z-d_{y y}(x, y) y_{1} y_{2} \\
& \left.z^{|W|}\right|_{\Omega}=0, \quad|W|=0,1,2, \cdots
\end{aligned}
$$

with this, the proof of the Theorem is complete.

\subsection{The Second Derivative of the Cost Functional}

Under the Assumption 2, the cost function $J$ is twice continuously Fréchet differentiable in $W^{\infty}\left\{a_{\alpha}, 2\right\} \times L^{\infty}(\Omega)$. By virtue of the chain rule $f: u \rightarrow$ $J(G(u, u))$ is then also twice continuously Fréchet differentiable in $L^{\infty}(\Omega)$. The second derivative can be calculated as in the preceding proof of Theorem 4.1. First, we obtain

$$
f^{\prime}(u) u_{1}=D_{y} J(G(u), u) G^{\prime}(u) u_{1}+D_{u} J(G(u), u) u_{1}
$$

Next, we calculate the direction derivative of $\tilde{f}(u)=f^{\prime}(u) u_{1}$ in the direction $u_{2}$. 
Invoking the product and chain rules, we find that

$$
\begin{aligned}
f^{\prime \prime}(u)\left[u_{1}, u_{2}\right]= & \tilde{f}^{\prime}(u) u_{2} \\
= & D_{y}^{2} J(G(u), u)\left[G^{\prime}(u) u_{1}, G^{\prime}(u) u_{2}\right]+D_{u} D_{y} J(G(u), u)\left[G^{\prime}(u) u_{1}, u_{2}\right] \\
& +D_{y} J(G(u), u) G^{\prime \prime}(u)\left[u_{1}, u_{2}\right]+D_{y} D_{u} J(G(u), u)\left[u_{1}, G^{\prime}(u) u_{2}\right] \\
& +D_{u}^{2} J(G(u), u)\left[u_{1}, u_{2}\right] \\
= & J^{\prime \prime}(y, u)\left[\left(y_{1}, u_{1}\right),\left(y_{2}, u_{2}\right)\right]+D_{y} J(u, u) G^{\prime \prime}(u)\left[u_{1}, u_{2}\right]
\end{aligned}
$$

To simplify the terms, we again use the abbreviations $z:=G^{\prime \prime}(u)\left[u_{1}, u_{2}\right]$ and $y_{i}:=G^{\prime}(u) u_{i} ; i=1,2$. With this, we obtain the expression

$$
D_{y} J(y, u) z=\int_{\Omega} \phi_{y}(x, y(x)) z(x) d x
$$

which can be easily transformed using the adjoint state $\rho$. In fact, $\rho$ is defined as the unique weak solution to the boundary value problem

$$
\begin{aligned}
& A p+d_{y}(., y) p=\phi_{y}(., y) \\
& \left.p^{|W|}\right|_{\Omega}=0 \quad|W|=0,1,2, \cdots
\end{aligned}
$$

By virtue of Theorem 4.1, $z$ solves the distributed problem (19) whose right hand side $\tilde{u}:=-d_{y y}(., y) y_{1} y_{2}$ can be regarded as a control. Specifying the involved quantities appropriately, we have

$$
D_{y} J(y, u) z=\int_{\Omega} p \tilde{u} d x=-\int_{\Omega} p d_{y y}(x, y) y_{1} y_{2} d x
$$

using this in (20) finally yields

$$
f^{\prime \prime}(u)\left[u_{1}, u_{2}\right]=J^{\prime \prime}(y, u)\left[\left(y_{1}, u_{1}\right),\left(y_{2}, u_{2}\right)\right]-\int_{\Omega} p d_{y y}(x, y) y_{1} y_{2} d x
$$

under our consideration and apply a Theorem of F. Tröltzsch [14] we have this Theorem.

Theorem 9. Let $U=L_{2}(\Omega)$ be the space of control, let $U_{a d} \subset U$ be convex and suppose that the functional $f: U \rightarrow R$ is twice continuously Frechet differentiable in an open neighborhood of $\bar{u} \in U_{a d}$. Let the control $\bar{u}$ satisfy the first-order necessary condition

$$
f^{\prime}(u)(u-\bar{u}) \geq 0 \quad \forall u \in U_{a d}
$$


and assume there exists a $\delta>0$ such that

$$
f^{\prime \prime}(\bar{u})[h, h] \geq \delta\|h\|^{2}
$$

then there exists constants $\epsilon>0$ and $\sigma>0$ so that

$$
f(u) \geq f(\bar{u})+\sigma\|u-\bar{u}\|_{L^{2}(\Omega)}^{2} \quad \forall u \in U_{a d}
$$

such that $\|u-\bar{u}\|_{L^{2}(\Omega)} \leq \epsilon$.

Thus, $\bar{u}$ is a local minimum for $f$ on $U_{a d}$.

Proof. The proof is the same as that in a finite-dimensional space, and we use the abbreviation $f^{\prime \prime}(\bar{u}) h^{2}:=f^{\prime \prime}(\bar{u})[h, h]$.

Consider the function $F:[0,1] \rightarrow R$,

$$
F(s):=f(\bar{u}+s(u, \bar{u})
$$

then $F(1)=f(u)$ and $F(0)=f(\bar{u})$. Using Taylor expansion

$$
F(1)=F(0)+F^{\prime}(0)+\frac{1}{2} F^{\prime \prime}(\theta), \quad \theta \in(0,1)
$$

we obtain

$$
\begin{aligned}
f(u) & =f(\bar{u})+f^{\prime}(u)(u-\bar{u})+\frac{1}{2} f^{\prime \prime}(\bar{u}+\theta(u-\bar{u})(u-\bar{u}, u-\bar{u}) \\
\geq & f(\bar{u})+\frac{1}{2} f^{\prime \prime}(\bar{u}+\theta(u-\bar{u})(u-\bar{u}, u-\bar{u}) \\
= & f(\bar{u})+\frac{1}{2} f^{\prime \prime}(\bar{u})(u-\bar{u}, u-\bar{u}) \\
& +\frac{1}{2} f^{\prime \prime}\left(\bar{u}+\theta(u-\bar{u})-f^{\prime \prime}(u)\right)(u-\bar{u}, u-\bar{u})
\end{aligned}
$$

Setting $h=u-\bar{u}$ in (23), we find that

$$
f^{\prime \prime}(\bar{u})(u-\bar{u})^{2} \geq \delta\|u-\bar{u}\|_{L^{2} \Omega}^{2} .
$$

Since $u \rightarrow f^{\prime \prime}(u)$ is continuous (in a neighborhood of $\bar{u}$ ) we conclude from (23)

$$
f(u) \geq f(\bar{u})+\frac{\delta}{2}\|u-\bar{u}\|_{L^{2} \Omega}^{2}-\frac{\delta}{4}\|u-\bar{u}\|_{L^{2} \Omega}^{2}
$$

provided $\|u-\bar{u}\|_{L^{2} \Omega} \leq \epsilon$. Thus

$$
f(u) \geq f(\bar{u})+\frac{\delta}{4}\|u-\bar{u}\|_{L^{2} \Omega}^{2}
$$

which given the claim for $\sigma=\frac{\delta}{4}$. 


\section{References}

[1] H. Cartan, Calcul Differentiel, Formes Différentielles, Hermann, Paris, 1967.

[2] E. Casas and F. Tröltzsch, First and second-order optimalty conditions for a class of optimal control problems with quasilinear elliptic equations, SIAM J. control optim., 48 (2009), 688-718.

[3] E. Casas and F. Tröltzsch, A general theorem on error estimates with application to a quasilinear elliptic optimal control problems, Computational Optimization and Applications, 53 (2012), 173-206.

[4] E. Casas and Mariano Mateos, Second order optimality conditions for semilinear elliptic control problems with finitely many state constraints, SIAM J. control optim., 40 (2002), 1431-1454.

[5] Ju. A. Dubinskii, Sobolev spaces of infinite order and the behavior of solutions of some boundary value problems with unbounded increase of the order of the equation, Math. USSR Sbornik, 27 (1975).

[6] Ju. A. Dubinskii, Some imbedding theorem for Sobloev spaces of inifinite order, Soviet Math. Dokl., 19 (1979), 1271-1274.

[7] S. A. El-Zahaby and GH. H. Mostafa, Optimal control of variational inequalities of infinite order, J. of Math. Egyptian Soc., 13 (2005), 99-106.

[8] S. A. El-Zahaby and El-Zoulali, Ibtissam, Optimal control of problems governed by obstacle type for infinite order, Proc. of the 8th International Scientific Conference Enviromment, Development and Bioinformations, Faculty of Science, Al-Azhar University, 26-28 march 2012, Nasr City, Cairo, Egypt.

[9] I. M. Gali, Optimal control of system governed by elliptic operators of infinite order, Ordinary and partial differential equations, Proceedings of the 1982 Dumdec Conference, Dundee, Lecture Notes in Mathematics, pp. 263-272, (1984).

[10] I. M. Gali, and H. A. El-Saify, Optimal control of a system governed by a self adjoint ellptic operator with an infinite number of variables, Proceeding of the International Conference on "Functional Differential Systems and Related Topics II", Poland, may 1991. 
[11] I. M. Gali, and H. A. El-Saify, Control of system governed by infinite order equations of hyperbolic type, Proceedings of Optimization Days, 11-13 May 1983, Montreal, Canada.

[12] I. M. Gali and S. A. El-Zahaby, Optimal control of a system governed by variational inequalities for infinite order elliptic operator, Kyngpook Mathematical Jounral, 31 (1991), 193-200.

[13] I. M. Gali, H. A. El-Saify and S. A. El-Zahaby, Distributed control of a system governed by Dirichlet and Neumann problems foe elliptic equations of infinite order, Proc. of the International conference of Functional Differential System and Related topics III, Pland, (1983), pp. 22-29.

[14] H. Goldberg and F. Tröltzsch, Second order optimality conditions for a class of control problems governed by non-linear intergral equations with applications to parabolic boundary control optimization 20, pp. 687-698, 1989.

[15] H. Goldberg and F. Tröltzsch, Second order sufficient optimality conditions for a class of nonlinear parabolic boundary control problems, SIAM J. control optim., 31 (1993), 1007-1027.

[16] F. Tröltzsch, Optimal control of partial differential equations. Theory, Methods and Applications, American Mathematical Society Provnedence, Rhode, Island, 2010. 